PROCEEDINGS OF THE

AMERICAN MATHEMATICAL SOCIETY

Volume 137, Number 11, November 2009, Pages 3741-3748

S 0002-9939(09)09928-6

Article electronically published on May 27, 2009

\title{
AN OPERATOR ALGEBRAIC PROOF OF AGLER'S FACTORIZATION THEOREM
}

\author{
SNEH LATA, MEGHNA MITTAL, AND VERN I. PAULSEN
}

(Communicated by Marius Junge)

\begin{abstract}
We give a short direct proof of Agler's factorization theorem that uses the Blecher-Ruan-Sinclair characterization of operator algebras. The key ingredient of this proof is an operator algebra factorization theorem. Our proof provides some additional information about these factorizations in the case of polynomials.
\end{abstract}

\section{INTRODUCTION}

Given an analytic function $f$ on the open unit disk $\mathbb{D}$, Nevanlinna proved that the supremum of $f$ over the disk is less than or equal to one if and only if the function $\frac{1-f(z) \overline{f(w)}}{1-z \bar{w}}$ is a positive definite function on $\mathbb{D}$. This latter condition is equivalent to the existence of a positive definite function $K: \mathbb{D}^{2} \rightarrow \mathbb{C}$ which is analytic in $z$ and coanalytic in $w$ such that $1-f(z) \overline{f(w)}=(1-z \bar{w}) K(z, w)$. Here we are following the standard usage of calling a function positive definite if for every choice of finitely many points, the matrix $\left(K\left(z_{i}, z_{j}\right)\right)$ is positive semidefinite. Later this result was generalized to a parallel result for analytic matrix-valued functions on the disk whose supremum norm is less than or equal to one.

A remarkable extension of this result to more than one variable was given by Agler [1. To explain his result we need to first introduce the Schur-Agler space of analytic functions on a polydisk. Given a natural number $N$ and $I=\left(i_{1}, \ldots, i_{N}\right) \in$ $\mathbb{N}^{N}$, we set $z^{I}=z_{1}^{i_{1}} \cdots z_{N}^{i_{N}}$ so that every analytic function $f: \mathbb{D}^{N} \rightarrow \mathbb{C}$ can be written as a power series, $f(z)=\sum_{I} a_{I} z^{I}$. If $T=\left(T_{1}, \ldots, T_{N}\right)$ is an $N$-tuple of operators on some Hilbert space $\mathcal{H}$ which pairwise commute and satisfy $\left\|T_{i}\right\|<1$ for $i=1, \ldots N$, then we will call $T$ a commuting $N$-tuple of strict contractions. It is easily seen that if $T$ is a commuting $N$-tuple of strict contractions, then the power series $f(T)=\sum_{I} a_{I} T^{I}$ converges and defines a bounded operator on $\mathcal{H}$. The SchurAgler space, denoted by $H_{u}^{\infty}\left(\mathbb{D}^{N}\right)$, is defined to be the set of analytic functions on $\mathbb{D}^{N}$ such that $\|f\|_{u}=\sup \{\|f(T)\|\}$ is finite, where the supremum is taken over all sets of commuting $N$-tuples of strict contractions and all Hilbert spaces. In fact, the same supremum is attained by restricting to all commuting $N$-tuples of strict contractions on a fixed separable infinite dimensional Hilbert space. It is fairly easy

Received by the editors July 9, 2008, and, in revised form, February 16, 2009.

2000 Mathematics Subject Classification. Primary 46L07; Secondary 47L25.

This research was supported in part by NSF grant DMS-0600191.

(C)2009 American Mathematical Society 
to see that $H_{u}^{\infty}\left(\mathbb{D}^{N}\right)$ is a Banach algebra in the norm $\|\cdot\|_{u}$. The set of functions with $\|f\|_{u} \leq 1$ is called the Schur-Agler class.

Given a vector space $V$, we let $M_{m, n}(V)$ denote the set of $m \times n$ matrices with entries from $V$. Given $F=\left(f_{i, j}\right) \in M_{m, n}\left(H_{u}^{\infty}\left(\mathbb{D}^{N}\right)\right)$, we set $\|F\|_{u}=\sup \left\{\left\|\left(f_{i, j}(T)\right)\right\|\right\}$, where the supremum is again over all commuting $N$-tuples of strict contractions and the norm of $\left(f_{i, j}(T)\right)$ is computed by regarding the operator matrix as an operator from the direct sum of $n$ copies of the Hilbert space to the direct sum of $m$ copies of the Hilbert space.

Note that when the Hilbert space is one-dimensional, then every commuting $\mathrm{N}$ tuple of strict contractions $T$ is of the form $T=z=\left(z_{1}, \ldots, z_{N}\right) \in \mathbb{D}^{N}$, so that $\|f\|_{\infty}=\sup \left\{|f(z)|: z \in \mathbb{D}^{N}\right\} \leq\|f\|_{u}$ and hence $H_{u}^{\infty}\left(\mathbb{D}^{N}\right) \subseteq H^{\infty}\left(\mathbb{D}^{N}\right)$, where this latter space denotes the set of bounded analytic functions on the polydisk $\mathbb{D}^{N}$. When $N=1$ or 2 it is known that these two spaces of functions are equal and that $\|F\|_{u}=\|F\|_{\infty}$, for all $m$ and $n$. This is a consequence of theorems of J. von Neumann [7, Sz.-Nagy [6] and Ando [3].

For $N \geq 3$, it is known that these two norms are not equal. However, it is still unknown, for a general $N \geq 3$, if these two Banach spaces define the same sets of functions, since by the bounded inverse theorem $H_{u}^{\infty}\left(\mathbb{D}^{N}\right)=H^{\infty}\left(\mathbb{D}^{N}\right)$ if and only if there is a constant $K_{N}$ such that $\|f\|_{u} \leq K_{N}\|f\|_{\infty}$. The existence of such a constant is a problem that has been open since the early 1960's. For more details on all of these ideas one can see Chapters 5 and 18 of [8. A note of caution: in [1] it is stated that $H_{u}^{\infty}\left(\mathbb{D}^{N}\right) \neq H^{\infty}\left(\mathbb{D}^{N}\right)$ for $N \geq 3$, but what is meant is that the norms are not equal, and the question of whether or not they are equal as sets is, indeed, still open.

Agler's factorization theorem [1] says that $F \in M_{m, n}\left(H_{u}^{\infty}\left(\mathbb{D}^{N}\right)\right)$ with $\|F\|_{u} \leq 1$ if and only if there exist positive definite matrix-valued functions $K_{i}: \mathbb{D}^{N} \times \mathbb{D}^{N} \rightarrow$ $M_{m}, i=1, \ldots, N$, which are analytic in the first variables and coanalytic in the second variables, such that $I_{m}-F(z) F(w)^{*}=\sum_{i=1}^{N}\left(1-z_{i} \overline{w_{i}}\right) K_{i}(z, w)$. When $m=n=N=1$, Agler's result reduces to Nevanlinna's factorization theorem since in that case $\|F\|_{\infty}=\|F\|_{u}$ by von Neumann's inequality [7. The book by Agler and McCarthy [2] is an excellent source for further information and background on this result.

In this paper we show that Agler's factorization result is a direct consequence of the factorization ideas of Blecher and the third author [4] arising from the abstract theory of operator algebras. Our proof has the advantages of being relatively short, assuming the abstract characterization of operator algebras [5], and of giving some possibly new information on the form of the positive definite functions $K_{i}$ that appear in the Agler factorization. Our proof uses an observation of S. McCullough that led to Theorem 4 .

\section{MAIN RESUlts}

We let $\mathcal{P}_{N} \subseteq H_{u}^{\infty}\left(\mathbb{D}^{N}\right)$ denote the subspace spanned by the polynomials in $N$ variables endowed with the $\|\cdot\|_{u}$ norm. The equivalence of (i) and (ii) in the following theorem is a reformulation of results found in [4] and in the book [8, Chapter 18], but we will sketch a proof for clarity. The equivalence of (i) and (iii) is a useful variant of Agler's factorization result for polynomials.

It will be convenient to say that matrices $A_{1}, \ldots A_{m}$ are of compatible sizes if the product $A_{1} \cdots A_{m}$ exists, that is, provided that each $A_{i}$ is an $n_{i} \times n_{i+1}$ matrix. 
Theorem 1. Let $P=\left(p_{i j}\right) \in M_{m, n}\left(\mathcal{P}_{N}\right)$. Then the following are equivalent:

(i) $\|P\|_{u}<1$.

(ii) There exist an integer $l$, matrices of scalars $C_{j}, 1 \leq j \leq l$, with $\left\|C_{j}\right\|<1$ and diagonal matrices $D_{j}, 1 \leq j \leq l$, each consisting of monomials in one of the variables $z_{i_{j}}$, which are of compatible sizes and are such that $P(z)=C_{1} D_{1}\left(z_{i_{1}}\right) \cdots C_{l} D_{l}\left(z_{i_{1}}\right)$.

(iii) There exist a positive, invertible matrix $R \in M_{m}$ and matrices of polynomials $P_{i}, 0 \leq i \leq N$, such that

$$
I_{m}-P(z) P(w)^{*}=R+P_{0}(z) P_{0}(w)^{*}+\sum_{i=1}^{N}\left(1-z_{i} \overline{w_{i}}\right) P_{i}(z) P_{i}(w)^{*}
$$

where $z=\left(z_{1}, \ldots, z_{N}\right), w=\left(w_{1}, \ldots, w_{N}\right) \in \mathbb{D}^{N}$.

Proof. The proof that (i) implies (ii) follows from either [4, Corollary 2.11] or [8. Corollary 18.2], once one observes that $D_{l}$ could be the identity matrix. To illustrate how the abstract characterization of operator algebras is involved, we outline a direct proof.

For each $m, n \in \mathbb{N}$, one proves that $\|P\|_{m, n}:=\inf \left\{\left\|C_{1}\right\| \ldots\left\|C_{l}\right\|\right\}$ defines a norm on $M_{m, n}\left(\mathcal{P}_{N}\right)$, where the infimum is taken over all $l$ and all ways of factorizing $P(z)=C_{1} D_{1}\left(z_{i_{1}}\right) \cdots C_{l} D_{l}\left(z_{i_{l}}\right)$ as a product of matrices of compatible sizes with scalar matrices $C_{j}, 1 \leq j \leq l$, and diagonal matrices $D_{j}, 1 \leq j \leq l$, whose diagonal entries are monomials in one of the variables $z_{i_{j}}$.

Moreover, one can verify that $\mathcal{M}_{m, n}\left(\mathcal{P}_{N}\right)$ with this family $\left\{\|\cdot\|_{m, n}\right\}_{m, n}$ of norms satisfies the axioms for an abstract unital operator algebra as given in 5 ; hence by the Blecher-Ruan-Sinclair representation theorem [5] (see also [8]) there exist a Hilbert space $\mathcal{H}$ and a unital completely isometric isomorphism $\pi: \mathcal{P}_{N} \longrightarrow$ $\mathcal{B}(\mathcal{H})$. Thus, for every $m, n \in \mathbb{N}$ and every $P=\left(p_{i j}\right) \in \mathcal{M}_{m, n}\left(\mathcal{P}_{N}\right)$, we have $\|P\|_{m, n}=\left\|\pi\left(p_{i j}\right)\right\|$. However, $\left\|\pi\left(z_{i}\right)\right\|=\left\|z_{i}\right\|_{1,1} \leq 1$, and so $T=\left(\pi\left(z_{1}\right), \ldots, \pi\left(z_{n}\right)\right)$ is a commuting $N$-tuple of contractions. Thus, $\|P\|_{m, n}=\left\|\left(\pi\left(p_{i j}\right)\right)\right\|=\left\|\left(p_{i j}(T)\right)\right\| \leq$ $\|P\|_{u}$. This completes the proof that (i) implies (ii).

We will now prove that (ii) implies (iii). Suppose that $P$ has a factorization as in (ii). We will use induction on $l$ to prove that (iii) holds.

First, assume that $l=1$ so that $P(z)=C_{1} D_{1}\left(z_{i_{1}}\right)$. Then,

$$
\begin{aligned}
I_{m}-P(z) P(w)^{*} & =I_{m}-\left(C_{1} D_{1}\left(z_{i_{1}}\right)\right)\left(C_{1} D_{1}\left(z_{i_{1}}\right)\right)^{*} \\
& =\left(I_{m}-C_{1} C_{1}^{*}\right)+C_{1}\left(I-D_{1}\left(z_{i_{1}}\right) D_{1}\left(w_{i_{1}}\right)^{*}\right) C_{1}^{*} .
\end{aligned}
$$

Since $D_{1}\left(z_{i_{1}}\right)$ is a diagonal matrix of monomials in $z_{i_{1}}$, the $(k, k)$-th diagonal entry of $I-D_{1}\left(z_{i_{1}}\right) D_{1}\left(w_{i_{1}}\right)^{*}$ is $1-z_{i_{1}}^{n_{k}^{1}} \overline{w_{i_{1}}} n_{k}^{1}$ for some $n_{k}^{1}$. Thus,

$$
\begin{aligned}
1-z_{i_{1}}^{n_{k}^{1}} \overline{w_{i_{1}}} n_{k}^{1} & =\left(1-z_{i_{1}} \overline{w_{i_{1}}}\right)\left(1+z_{i_{1}} \overline{w_{i_{1}}}+\cdots+z_{i_{1}}^{n_{k}^{1}-1} \overline{w_{i_{1}}} n_{k}^{1}-1\right. \\
& =\left(1-z_{i_{1}} \overline{w_{i_{1}}}\right) A_{k}^{1}\left(z_{i_{1}}\right) A_{k}^{1}\left(w_{i_{1}}\right)^{*},
\end{aligned}
$$

where $A_{k}^{1}\left(z_{i_{1}}\right)=\left(1, z_{i_{1}}, z_{i_{1}}^{2}, \ldots, z_{i_{1}}^{n_{k}^{1}-1}\right)$ is a $1 \times n_{k}^{1}$ matrix of monomials in $z_{i_{1}}$. Hence,

$$
C_{1}\left(1-D_{1}\left(z_{i_{1}}\right) D_{1}\left(w_{i_{1}}\right)^{*}\right) C_{1}^{*}=\left(1-z_{i_{1}} \overline{w_{i_{1}}}\right) C_{1} A_{1}\left(z_{i_{1}}\right) A_{1}\left(w_{i_{1}}\right)^{*} C_{1}^{*},
$$

where $A_{1}\left(z_{i_{1}}\right)$ is the direct sum of the matrices $A_{k}^{1}\left(z_{i_{1}}\right)$. Therefore,

$$
I_{m}-P(z) P(w)^{*}=R_{0}+\left(1-z_{i_{1}} \overline{w_{i_{1}}}\right) P_{i_{1}}(z) P_{i_{1}}(w)^{*},
$$


where $R_{0}=I_{m}-C_{1} C_{1}^{*}$ is a positive, invertible matrix and $P_{i_{1}}(z)=C_{1} A_{1}\left(z_{i_{1}}\right)$, so the form (iii) holds when $l=1$.

We now assume that the form (iii) holds for any $P(z)$ that has a factorization of length at most $l-1$, and we also assume that

$$
P(z)=C_{1} D_{1}\left(z_{i_{1}}\right) \cdots D_{l-1}\left(z_{i_{l-1}}\right) C_{l} D_{l}\left(z_{i_{l}}\right)=C_{1} D_{1}\left(z_{i_{1}}\right) P_{l-1}(z),
$$

where $P_{l-1}(z)$ has a factorization of length $l-1$.

Note that a sum of expressions like those on the right hand side of (iii) is again such an expression. This follows from using the fact that given any two matrices $A$ and $B$, we can write

$$
A A^{*}+B B^{*}=C C^{*}
$$

where $C=(A B)$.

Thus, it will be sufficient to show that $I_{m}-P(z) P(w)^{*}$ is a sum of expressions as above. To this end we have that

$$
\begin{aligned}
I_{m}-P(z) P(w)^{*}=\left(I_{m}-\right. & \left.C_{1} D_{1}\left(z_{i_{1}}\right) D_{1}\left(w_{i_{1}}\right)^{*} C_{1}^{*}\right) \\
& +\left(C_{1} D_{1}\left(z_{i_{1}}\right)\right)\left(I-P_{l-1}(z) P_{l-1}(w)^{*}\right)\left(D_{1}\left(w_{i_{1}}\right)^{*} C_{1}^{*}\right) .
\end{aligned}
$$

The first term of the above equation is of the form as on the right hand side of (iii) by the $l=1$ case. Also, the quantity $\left(I-P_{l-1}(z) P_{l-1}(w)^{*}\right)$ is of this form by the inductive hypothesis. Finally, note that if $H(z, w)$ can be written as such a sum, then so can $Q(z) H(z, w) Q(w)^{*}$; it then follows that the second term is also of the required form.

Finally, we will prove that (iii) implies (i). Suppose (iii) holds. Then for any commuting $N$-tuple of strict contractions $T=\left(T_{1}, \ldots, T_{N}\right)$, by expanding both sides in powers of $T^{I}\left(T^{*}\right)^{J}$ and comparing the coefficients of the power series, we see that

$$
I_{m}-P(T) P(T)^{*}=R+P_{0}(T) P_{0}(T)^{*}+\sum_{i=1}^{N} P_{i}(T)\left(I-T_{i} T_{i}^{*}\right) P_{i}(T)^{*} .
$$

Clearly, each term on the right hand side of the above inequality is positive, and since $R$ is strictly positive, say $R \geq \delta I_{m}$ for some scalar $\delta>0$, we have that $I_{m}-P(T) P(T)^{*} \geq \delta I_{m}$. Therefore $(1-\delta) I_{m} \geq P(T) P(T)^{*}$, which implies that $\|P(T)\| \leq \sqrt{1-\delta}$. Thus, since $T$ was arbitrary, $\|P\|_{u} \leq \sqrt{1-\delta}<1$, which proves (i).

Corollary 2. Let $P=\left(p_{i j}\right) \in M_{m, n}\left(\mathcal{P}_{N}\right)$. If $\|P\|_{u}<1$, then there exist positive definite matrix-valued functions $K_{i}: \mathbb{D}^{N} \times \mathbb{D}^{N} \rightarrow M_{m}, 0 \leq i \leq N$, whose components are polynomials such that

$$
I_{m}-P(z) P(w)^{*}=K_{0}(z, w)+\sum_{i=1}^{N}\left(1-z_{i} \overline{w_{i}}\right) K_{i}(z, w),
$$

for all $z, w \in \mathbb{D}^{N}$.

Proof. Using the form in (iii), we set $K_{0}(z, w)=R+P_{0}(z) P_{0}(w)^{*}$ and $K_{i}(z, w)=$ $P_{i}(z) P_{i}(w)^{*}$ for $1 \leq i \leq N$.

One of the advantages of the above factorization over Agler's form is that, since each positive definite function $K_{i}$ consists of polynomials, the associated reproducing kernel Hilbert spaces will be finite dimensional spaces of $\mathbb{C}^{m}$-valued polynomials. 
To see the connection between the factorization occurring in our (iii) and Agler's factorization, note that each of the terms $K_{i}(z, w)=P_{i}(z) P_{i}(w)^{*}, i \geq 2$, is a positive definite matrix-valued function that is analytic in the $z$ variables and coanalytic in the $w$ variables. If we set

$$
K_{1}(z, w)=\frac{R+P_{0}(z) P_{0}(w)^{*}}{1-z_{1} \bar{w}_{1}}+P_{1}(z) P_{1}(w)^{*}
$$

then $K_{1}(z, w)$ is also a positive definite matrix-valued function that is analytic in $z$ and coanalytic in $w$ and we have that $I_{m}-P(z) P(w)^{*}=\sum_{i=1}^{N}\left(1-z_{i} \bar{w}_{i}\right) K_{i}(z, w)$. Unfortunately, when written in this form, the reproducing kernel Hilbert space associated with this new function $K_{1}$ will generally be an infinite dimensional Hilbert space of analytic $\mathbb{C}^{m}$-valued functions, even though $P(z)$ was only polynomial valued.

To pass from the above theorem for polynomials to the full version of Agler's theorem, we need to first show that functions in $M_{m, n}\left(H_{u}\left(\mathbb{D}^{N}\right)\right)$ have nice approximations by matrices of polynomials. Note that if we write a matrix of analytic functions $F \in M_{m, n}\left(H_{u}\left(\mathbb{D}^{N}\right)\right), F=\left(f_{i, j}\right)$, as a power series $F(z)=\sum_{I} A_{I} z^{I}$, where $A_{I} \in M_{m, n}$ are scalar matrices, then for any commuting $N$-tuple of strict contractions we will have that $F(T)=\sum A_{I} \otimes T^{I}$, where by the tensor product of an $m \times n$ scalar matrix $B=\left(b_{i, j}\right)$ and an operator $R \in B(\mathcal{H})$ we mean the operator $B \otimes R=\left(b_{i, j} R\right)$ from the direct sum of $n$ copies of $\mathcal{H}$ to the direct sum of $m$ copies of $\mathcal{H}$.

The following fact is also used in Agler's proof [1].

Lemma 3. Given any factorization of the form $I_{m}-F(z) F(w)^{*}=\sum_{i=1}^{N}(1-$ $\left.z_{i} \bar{w}_{i}\right) K_{i}(z, w)$ in Agler's theorem, each of the functions $K_{i}(z, w)$ satisfies $\left\|K_{i}(z, w)\right\|^{2} \leq \frac{1}{\left(1-\left|z_{i}\right|^{2}\right)\left(1-\left|w_{i}\right|^{2}\right)}$ and hence is locally bounded on $\mathbb{D}^{N}$.

Proof. For $z=\left(z_{1}, \ldots, z_{N}\right) \in \mathbb{D}^{N}$, we have that

$$
\left(1-\left|z_{i}\right|^{2}\right) K_{i}(z, z) \leq I_{m}-F(z) F(z)^{*} \leq I_{m}
$$

hence $\left\|K_{i}(z, z)\right\| \leq \frac{1}{1-\left|z_{i}\right|^{2}}$.

As each $K_{i}$ is positive definite, using the positivity of the $2 \times 2$ block matrix corresponding to the pair of points $z, w \in \mathbb{D}^{N}$, we obtain

$$
\left\|K_{i}(z, w)\right\|^{2} \leq\left\|K_{i}(z, z)\right\| \cdot\left\|K_{i}(w, w)\right\| \leq \frac{1}{\left(1-\left|z_{i}\right|^{2}\right)\left(1-\left|w_{i}\right|^{2}\right)} .
$$

This inequality shows that $\left\|K_{i}(z, w)\right\|$ is bounded on $\left(r \mathbb{D}^{N}\right) \times\left(r \mathbb{D}^{N}\right)$ for any $0<r<1$ and hence is locally bounded.

Theorem 4. Take $F \in \mathcal{M}_{m, n}\left(H_{u}^{\infty}\left(\mathbb{D}^{N}\right)\right)$ with $F(z)=\sum_{I} A_{I} z^{I}$ for $z \in \mathbb{D}^{N}$. Then the sequence $\left\{P_{n}\right\}$ of matrices of polynomials $P_{n}(z)=\sum_{|I| \leq n}\left(1-\frac{|I|}{n+1}\right) A_{I} z^{I}$ converges locally uniformly to $F$ with $\left\|P_{n}\right\|_{u} \leq\|F\|_{u}$ for each $n$. Conversely, if there is a sequence $\left\{P_{n}\right\}$ of matrices of polynomials converging to $F$ pointwise on $\mathbb{D}^{N}$ with $\left\|P_{n}\right\|_{u} \leq 1$ for each $n$, then $\|F\|_{u} \leq 1$.

Proof. Fix an $n \in \mathbb{N}$ and consider the Fejer kernel,

$$
F_{n}(\theta)=\frac{1}{n+1} \sum_{k, l=0}^{n} e^{i(k-l) \theta} \text { for } \theta \in[0,2 \pi] \text {. }
$$


Note that for each fixed $z \in \mathbb{D}^{N}$ the function $\theta \mapsto F\left(z e^{i \theta}\right)=F\left(z_{1} e^{i \theta}, \ldots, z_{N} e^{i \theta}\right)$ is continuous. We define $P_{n}(z)=\frac{1}{2 \pi} \int_{0}^{2 \pi} F\left(z e^{i \theta}\right) F_{n}\left(e^{i \theta}\right) d \theta \quad$ for $z \in \mathbb{D}^{N}$, where the integration is in the Riemann sense. A direct calculation shows that $P_{n}(z)=$ $\sum_{|I| \leq n}\left(1-\frac{|I|}{n+1}\right) A_{I} z^{I}$, where $|I|=i_{1}+\cdots+i_{N}$.

Next check that for a fixed commuting $N$-tuple $T=\left(T_{1}, \ldots, T_{N}\right)$ of strict contractions on a Hilbert space $\mathcal{H}$, the map $\theta \mapsto F\left(T_{1} e^{i \theta}, \ldots, T_{N} e^{i \theta}\right)$ is continuous from the interval into $B(\mathcal{H})$ equipped with the norm topology. This follows from the fact that since we are dealing with strict contractions, $F\left(T e^{i \theta}\right)$ is a norm limit of partial sums of its power series. It now follows that

$$
P_{n}(T)=\frac{1}{2 \pi} \int_{0}^{2 \pi} F\left(T e^{i \theta}\right) F_{n}\left(e^{i \theta}\right) d \theta,
$$

where the integration is again in the Riemann sense.

Thus,

$$
\left\|P_{n}(T)\right\| \leq \frac{1}{2 \pi} \int_{0}^{2 \pi}\left\|F\left(T e^{i \theta}\right)\right\| F_{n}\left(e^{i \theta}\right) d \theta \leq\|F\|_{u},
$$

and we have shown that $\left\|P_{n}\right\|_{u} \leq\|F\|_{u}$.

The fact that $P_{n}$ converges to $F$ locally uniformly is a standard result for scalarvalued functions. To see this directly in our case, note that for $z \in \mathbb{D}^{N}$ we have that

$$
P_{n}(z)=\sum_{|I| \leq n} \frac{(n+1-|I|)}{n+1} A_{I} z^{I}=\frac{S_{0}(z)+\cdots+S_{n}(z)}{n+1},
$$

where $S_{k}(z)=\sum_{|I| \leq k} A_{I} z^{I}$ for $k=1, \ldots, n$, and hence $P_{n} \rightarrow F$ locally uniformly on $\mathbb{D}^{N}$.

For the converse, let $\left\{P_{n}\right\}$ be a sequence of $\mathcal{M}_{m, n}$-valued polynomials with $\left\|P_{n}\right\|_{u} \leq 1$ converging to $F$ pointwise on $\mathbb{D}^{N}$. For each $n,\left\|P_{n}\right\|_{\infty} \leq\left\|P_{n}\right\|_{u} \leq 1$. This implies that there exists a subsequence $\left\{P_{n_{k}}\right\}$ which converges to a function $G \in \mathcal{M}_{m, n}\left(H^{\infty}\left(\mathbb{D}^{N}\right)\right)$ in the weak* topology and, hence, that $\left\{P_{n_{k}}\right\}$ converges to $G$ uniformly on compact subsets of $\mathbb{D}^{N}$. Thus, $G=F$ and $\left\{P_{n_{k}}\right\}$ converges to $F$ uniformly on compact subsets of $\mathbb{D}^{N}$. If we now take $T=\left(T_{1}, \ldots, T_{N}\right)$ to be a commuting $N$-tuple of strict contractions, then there is an $r<1$ such that $\left\|T_{i}\right\| \leq r$ for all $i$. Since the polynomials converge to $F$ uniformly on the closed polydisk of radius $\frac{1+r}{2}$, it follows by the Riesz functional calculus that $P_{n_{k}}\left(T_{1}, \ldots, T_{N}\right) \longrightarrow F\left(T_{1}, \ldots, T_{N}\right)$ in norm.

Therefore, $\|F(T)\|=\lim _{k \rightarrow \infty}\left\|P_{n_{k}}(T)\right\| \leq 1$ and, hence, $\|F\|_{u} \leq 1$.

We can now prove Agler's theorem.

Theorem 5 (Agler's Factorization Theorem). Let $F \in \mathcal{M}_{m, n}\left(\mathcal{H}^{\infty}\left(\mathbb{D}^{N}\right)\right)$. Then $\|F\|_{u} \leq 1$ if and only if there exist positive definite functions $K_{i}: \mathbb{D}^{N} \times \mathbb{D}^{N} \rightarrow$ $M_{m}, 1 \leq i \leq N$, analytic in the first variable and coanalytic in the second variable, such that

$$
I_{m}-F(z) F(w)^{*}=\sum_{i=1}^{N}\left(1-z_{i} \overline{w_{i}}\right) K_{i}(z, w),
$$

where $z=\left(z_{1}, \ldots, z_{N}\right), w=\left(w_{1}, \ldots, w_{N}\right) \in \mathbb{D}^{N}$.

Proof. Let $\|F\|_{u} \leq 1$. By Theorem 4, there exists a sequence $\left\{P_{n}\right\}$ of matrices of polynomials such that $P_{n}$ converges to $F$ locally uniformly on $\mathbb{D}^{N}$ with $\left\|P_{n}\right\|_{u}<1$ for each $n$. 
Since $\left\|P_{n}\right\|_{u}<1$, by Theorem 1 and the remarks following it, there exist positive definite functions $K_{i}^{n}: \mathbb{D}^{N} \times \mathbb{D}^{N} \rightarrow M_{m}, 1 \leq i \leq N$, analytic in the first variable and coanalytic in the second, such that

$$
I_{m}-P_{n}(z) P_{n}(w)^{*}=\sum_{i=1}^{N}\left(1-z_{i} \overline{w_{i}}\right) K_{i}^{n}(z, w),
$$

where $z=\left(z_{1}, \ldots, z_{N}\right)$ and $w=\left(w_{1}, \ldots, w_{N}\right)$.

Moreover, by Lemma 3, for each $(z, w) \in \mathbb{D}^{N} \times \mathbb{D}^{N}$ and for each $n$ and $i$, $\left\|K_{i}^{n}(z, w)\right\|^{2} \leq \frac{1}{\left(1-\left|z_{i}\right|^{2}\right)\left(1-\left|w_{i}\right|^{2}\right)}$. So, for each fixed $i \in\{1, \ldots, N\}$ we have a locally bounded sequence $\left\{K_{i}^{n}: n \in \mathbb{N}\right\}$ of analytic-coanalytic functions. Thus, each of the $m^{2}$ functions that make up the coefficients of the matrix-valued functions $K_{i}^{n}: \mathbb{D}^{N} \times \mathbb{D}^{N} \longrightarrow M_{m}$ is locally bounded.

Hence, applying Montel's theorem $\left(m^{2} N\right.$ times $)$, there exist a subsequence $\left\{K_{i}^{n_{k}}\right\}$ and analytic-coanalytic functions $K_{i}: \mathbb{D}^{N} \times \mathbb{D}^{N} \rightarrow M_{m}, 1 \leq i \leq N$, such that the subsequence $\left\{K_{i}^{n_{k}}\right\}$ converges to $K_{i}$ locally uniformly on $\mathbb{D}^{N} \times \mathbb{D}^{N}$. Since each function in the subsequence is positive definite, it follows that each $K_{i}$ is positive definite. Taking limits, we have that

$$
I_{m}-F(z) F(w)^{*}=\lim _{k}\left(I_{m}-P_{n_{k}}(z) P_{n_{k}}(w)^{*}\right)=\sum_{i=1}^{N}\left(1-z_{i} \bar{w}_{i}\right) K_{i}(z, w) .
$$

The proof of the converse is identical to the argument given by Agler [1]. We briefly recall it for completeness. Assume that we are given that

$$
I_{m}-F(z) F(w)^{*}=\sum_{i=1}^{N}\left(1-z_{i} \overline{w_{i}}\right) K_{i}(z, w)
$$

where each $K_{i}$ is a positive definite $M_{m}$-valued function that is analytic in $z$ and coanalytic in $w$.

From the general theory of reproducing kernel Hilbert spaces, it follows that there exist separable Hilbert spaces $\mathcal{H}_{i}, 1 \leq i \leq N$, and analytic functions $F_{i}$ : $\mathbb{D}^{N} \rightarrow B\left(\mathcal{H}_{i}, \mathbb{C}^{m}\right), 1 \leq i \leq N$, such that $K_{i}(z, w)=F_{i}(z) F_{i}(w)^{*}$ for $1 \leq i \leq N$. For those unfamiliar with this theory, let $\mathcal{H}_{i}$ be the reproducing kernel Hilbert space of $\mathbb{C}^{m}$-valued functions constructed from $K_{i}$ and let $F_{i}(z): \mathcal{H}_{i} \rightarrow \mathbb{C}^{m}$ be evaluation at $z$. Choosing an orthonormal basis for $\mathcal{H}_{i}$, one may regard $F_{i}(z)$ as an $m \times \infty$ matrix of analytic functions on $\mathbb{D}^{N}$. One then verifies that for any commuting $N$-tuple of strict contractions,

$$
I_{m}-F(T) F(T)^{*}=\sum_{i=1}^{N} F_{i}(T)\left(I-T_{i} T_{i}^{*}\right) F_{i}(T)^{*} \geq 0,
$$

and so $\|F(T)\| \leq 1$. Since $T$ was arbitrary, we have that $\|F\|_{u} \leq 1$.

As we showed in the above proof, each analytic-coanalytic positive definite function $K_{i}: \mathbb{D}^{N} \times \mathbb{D}^{N} \rightarrow M_{m}$ can be factored as $K_{i}(z, w)=F_{i}(z) F_{i}(w)^{*}$, where $F_{i}$ is an $m \times \infty$ matrix of analytic functions on $\mathbb{D}^{N}$. Often Agler's factorization theorem is written in this equivalent form, i.e., as

$$
I_{m}-F(z) F(w)^{*}=\sum_{i=1}^{N}\left(1-z_{i} \overline{w_{i}}\right) F_{i}(z) F_{i}(w)^{*} .
$$




\section{REFERENCES}

[1] J. Agler, On the Representation of Certain Holomorphic Functions Defined on a Polydisk, Topics in Operator Theory: Ernst D. Hellinger Memorial Volume, Oper. Theory Adv. Appl., 48(1990), Birkhäuser, Basel, 47-66. MR1207393 (93m:47013)

[2] J. Agler and J. E. McCarthy, Pick Interpolation and Hilbert Function Spaces, Graduate Studies in Mathematics, 44, American Mathematical Society, Providence, RI, 2002. MR 1882259 (2003b:47001)

[3] T. Ando, On a Pair of Commutative Contractions, Acta Sci. Math., 24(1963), 88-90. MR0155193 (27:5132)

[4] D. P. Blecher and V. I. Paulsen, Explicit Constructions of Universal Operator Algebras and Applications to Polynomial Factorization, Proc. Amer. Math. Soc., 112(1991), 839-850. MR.1049839 (91j:46093)

[5] D. P. Blecher, Z. J. Ruan and A. M. Sinclair, A Characterization of Operator Algebras, J. Funct. Anal., 89(1990), 188-201. MR.1040962 (91b:47098)

[6] B. Sz-Nagy, Sur les contractions de l'espace de Hilbert, Acta. Sci. Math., 15(1953), 87-92. MR.0058128 (15:326d)

[7] J. von Neumann, Eine Spektraltheorie fur allgemeine Operatoren eines unitaren Raumes, Math. Nachr., 4(1951), 258-281. MR0043386 (13:254a)

[8] V. I. Paulsen, Completely Bounded Maps and Operator Algebras, Cambridge Studies in Advanced Mathematics, 78, Cambridge University Press, Cambridge, 2002. MR 1976867 (2004c:46118)

Department of Mathematics, University of Houston, Houston, Texas 77204-3476

E-mail address: snehlata@math.uh.edu

Department of Mathematics, University of Houston, Houston, Texas 77204-3476

E-mail address: mittal@math.uh.edu

Department of Mathematics, University of Houston, Houston, Texas 77204-3476

E-mail address: vern@math.uh.edu 